\title{
The Use of Fibula Free Flap for Reconstruction of Anterior Chest Wall Full-Thickness Defects
}

\section{Szumniak Ryszard, Maciejewski Adam}

Maria Sklodowska-Curie Memorial Cancer Center and Institute of Oncology, The Oncologic and Reconstructive Surgery Clinic, Gliwice Branch, Gliwice, Poland.

Email: rszumniak@wp.pl, adammac@02.pl

Received June $18^{\text {th }}, 2013$; revised July $18^{\text {th }}, 2013$; accepted July $25^{\text {th }}, 2013$

Copyright (C) 2013 Szumniak Ryszard, Maciejewski Adam. This is an open access article distributed under the Creative Commons Attribution License, which permits unrestricted use, distribution, and reproduction in any medium, provided the original work is properly cited. In accordance of the Creative Commons Attribution License all Copyrights (C) 2013 are reserved for SCIRP and the owner of the intellectual property Szumniak Ryszard, Maciejewski Adam. All Copyright (C) 2013 are guarded by low and by SCIRP as a guardian.

\begin{abstract}
Reconstructions of the sternum remain a formidable challenge for the operating team. Reconstruction of the sternumdefect, regardless of the reason, should ensure the stability of the anterior chest wall, the return of acceptable respiratory parameters and the control of wounds. Stability of the chest wall can be provided by autogenous tissues or prosthetic materials. In our experience, the fibula free osteocutaneous flaps are harvested for reconstruction of the bone defect in two patients after full-thickness defect of the sternum and anterior chest wall.
\end{abstract}

Keywords: Fibula Free Flap; Sternal Reconstruction; Sternotomy Wound Dehiscence; Recurrent Carcinoma

\section{Introduction}

The fibula flap has become one of the most commonly used vascularized osseous and osteocutaneous flaps for reconstruction of defects around the body, particularly of the mandible, radius, femur, and tibia. It can be osteotomized into multiple segments of differing lengths and shapes. The good vascularity of the bone segments is manifested. Various pathologic processes can affect the integrity of the chest wall. Stability of the thorax is required for physiological respiratory function. When the defect is large and/or complex, rigid support is necessary. Reconstruction of the chest wall can involve both skeletal and soft-tissue components. One option for reconstruction of the chest wall skeleton is autogenous bone grafts, which avoids the use of foreign materials. Donor sites for bone grafts include the ribs, iliac crest, and fibula. For successful reconstruction, the bone graft must be apposed to a large surface area of trabecular bone around the chest wall defect margins, so as to enhance graft survival and osteosynthesis. In this report, we present a technique for the reconstruction of extended 3D composite anterior chest wall defects using fibula free osteocutaneous flap in two patients.

\section{Case 1}

A 65-year-old male patient admitted to the Department of Oncological and Reconstructive Surgery with local advanced recurrent basal cell carcinoma infiltrating the upper sternal region. In the previous year, he had surgery to remove advanced skin cancer of sternum region with free anterolateral thigh flap reconstruction (Figure 1). It was painless, with no other associated symptoms like itching or bleeding. After 9 months, the recurrence was diagnosed based on CT and MRI and it involved the skin over the upper sternal region. The bone scan showed infiltration of the bone of the upper sternum and partialboth clavicles. Lesion was excised completely with wide margins all around. A defect of $14 \mathrm{~cm} \times 10 \mathrm{~cm}$ was created.Twoosteocutaneousfibula free flaps (bone segment $16 \mathrm{~cm}$; skin paddle $9 \times 12 \mathrm{~cm}$ ) was harvested for reconstruction of the sternum and clavicles and the part of the skin defect (Figure 2). The transverse artery was used as the recipient artery on both sides, and a vein was anastomosed withbranches of jugular vein.After osteotomy performed osteosynthesis using titanium plates (Figures 2 and 3). Skin paddles recreated defect of the skin over sternum (Figure 4). 


\section{Case 2}

A 62-year-old diabetic male were presented to our department with swelling, redness, and purulent discharge from a previous sternotomy site. He had undergone coronary artery bypass grafting 2 months earlier using left internal thoracic artery for silent ischemia. During the initial hospitalization he suffered from deep sternal wound infection following the coronary surgery, which was treated by debridement and a vacuum suctioning system followed by sternal revision using the Robicsek technique (Figure 5). For the treatment, surgical debridement and sternal reconstruction was planned. During surgery, the osteolytic lower manubrium and upper sternal body (from the lower margin of the first rib level to the upper margin of the fourth rib level) were removed and only the healthy part of sternum remained. Sternal stability was restored by osteocoutaneous fibula free flapand by three horizontal titanium plates. Each titanium plate was fixed to the corresponding rib using three titanium screws for each side (Figure 6). The osteocoutaneous fibula free flap was harvested from left leg (bone length 17, $6 \mathrm{~cm}$; skin paddle $16 \times 7 \mathrm{~cm}$ ) (Figure 7). Microvascular anastomoses were performed before rigid fixation of the bone graft. The flap transfer was performed with microvascular anastomoses of the artery and vein to the right internal mammary artery (IMA) using 9-0 and 10-0 Ethilon sutures (in this case, the left internal mammary artery (IMA) was previously harvested for a coronary artery bypass graft) (Figures 8 and 9). The anoxia time of the flap was 2 hours and the procedure took 8 hours. Osteotomy of the bone was necessary because the fibula bone could not simulate the curvature of the chest cage and thus avoid compression of the heart. A postoperative course was uneventful in both cases and follow-up CT showed no signs of infection in the sternum or mediastinum and also revealed that the chest wall integrity was well maintained (Figures 7 and 10). Postoperative

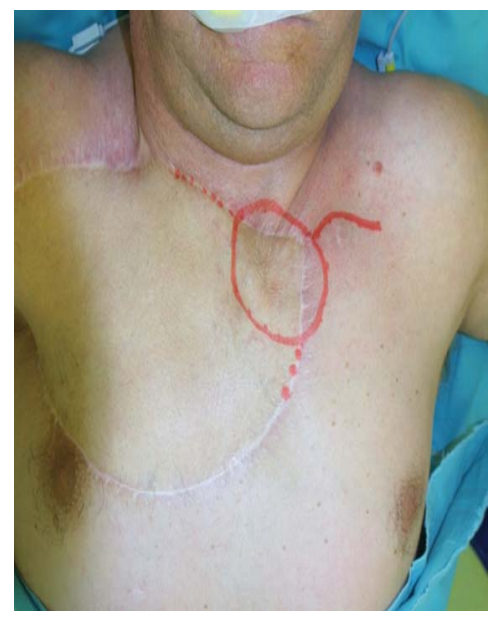

Figure 1. Preoperative view.

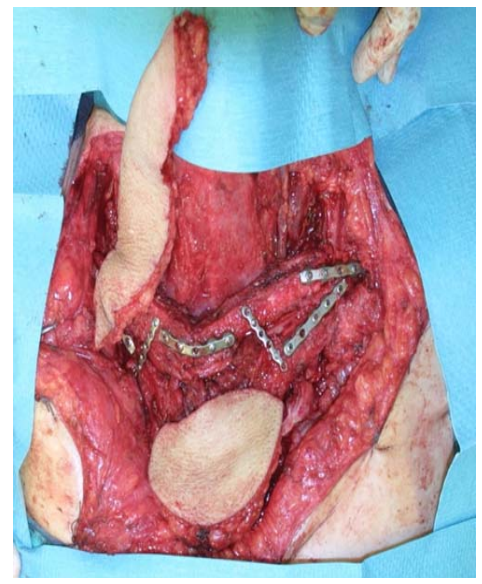

Figure 2. Implantation of fibula segments.

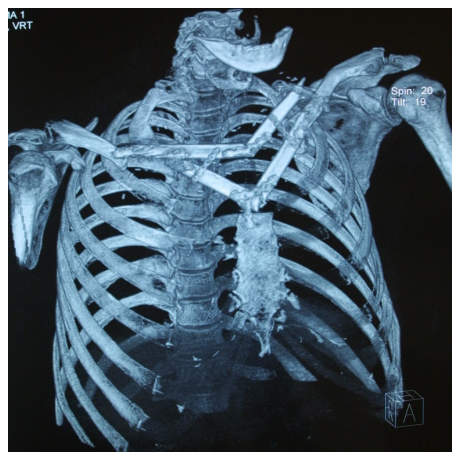

Figure 3. CT scan showing the fibula segments in position.

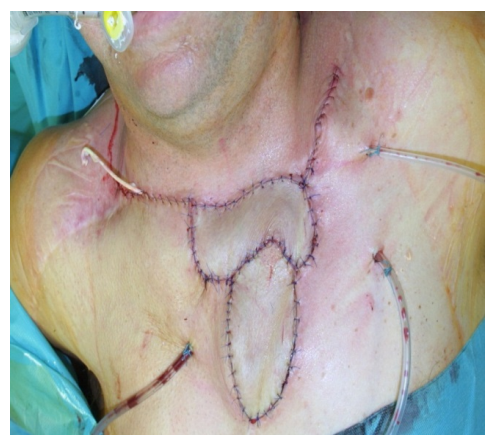

Figure 4. Early postoperative result.

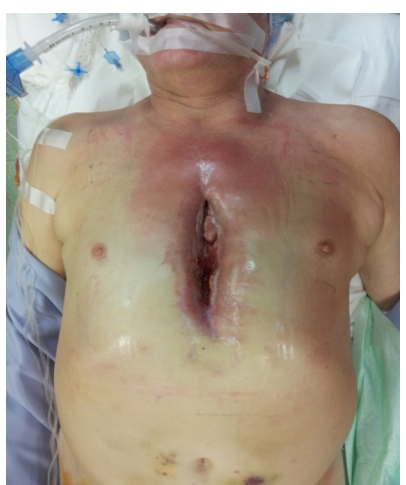

Figure 5. Preoperative condition. 


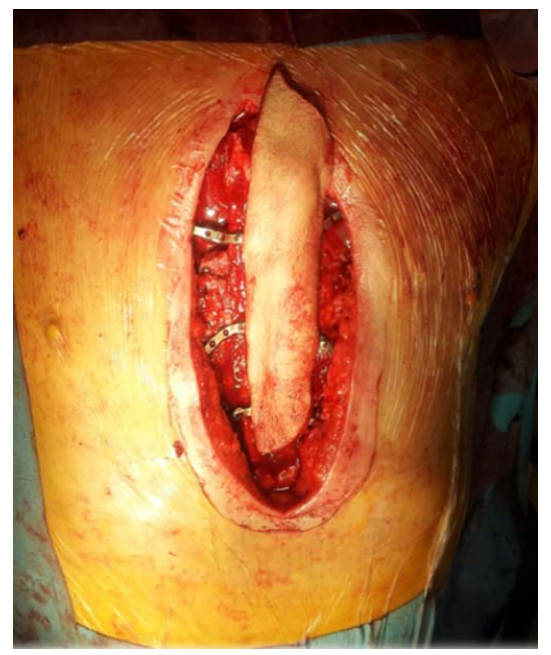

Figure 6. Intraoperative view after osteosynthesis.

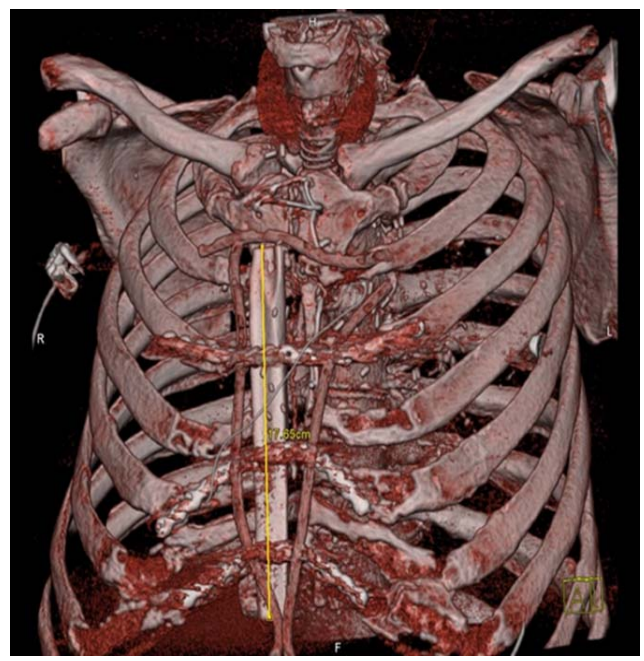

Figure 7. CT scan after implantation fibula and osteosynthesis.

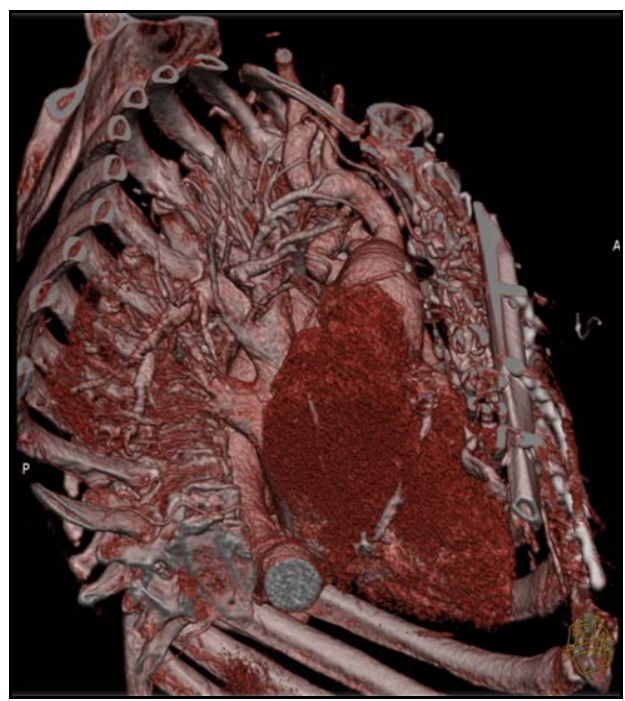

Figure 8. Postoperative angio-CT.

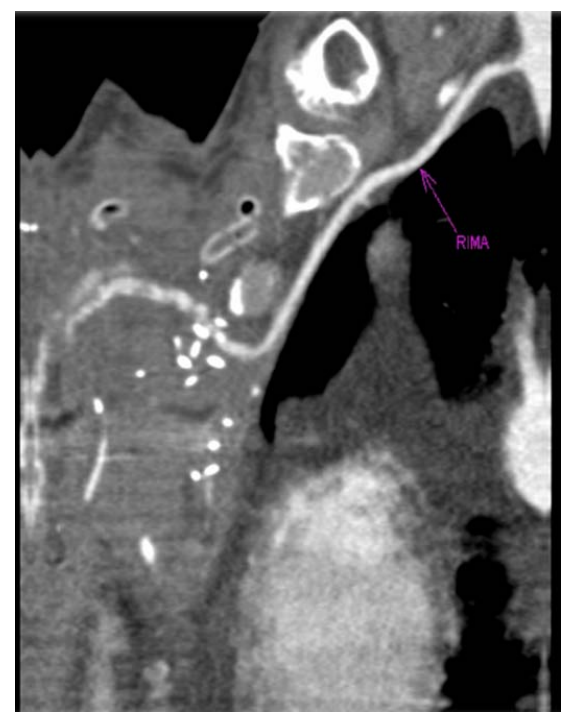

Figure 9. MRI showing place of microanastomosis.

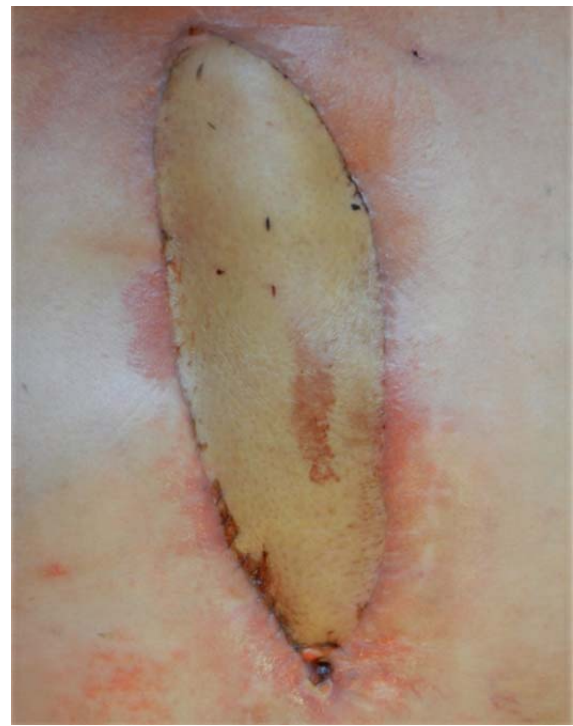

Figure 10. Postoperative result.

chest roentgenograms showed correct position of the bone grafts. During the 6-month follow-up period after surgery, both patients were doing well without any sternal wound problems and with no sign of recurrence disease.

\section{Discussion}

Full-thickness chest wall resection with reconstruction is still one of the most challenging operations today [1]. Various techniques have been applied to the reconstruction of the chest wall following resection. Nowadays, in chest wall reconstruction prosthetic materials like prosthetic mesh with methyl methacrylate are generally used. However, the rejections of prosthetic materials and infections frequently occur in chest wall reconstruction, espe- 
cially after postoperative radiotherapy or resection that is performed due to infections [1]. With a wide variety of etiologies of chest wall defects and varying degrees of complexity, the decision-making in reconstruction can be complicated. Successful reconstruction requires close collaboration between the reconstructive and thoracic surgeon. Multiple factors dictate the type of reconstruction undertaken, including the etiology of the defect; the patient's history, physical stability, hemodynamic status, ventilatory capacity, and comorbid conditions; and the ultimate prognosis. The specific chest wall defect should be evaluated in a three-dimensional fashion prior to reconstruction planning. One option for reconstruction of the chest wall skeleton is autogenous bone grafts, which avoids the use of foreign materials. Donor sites for bone grafts include the ribs, iliac crest, and fibula.

Sternal dehiscence is an infrequent but serious complication that is often a precursor to mediastinitis. Median sternotomy complications occur in $0.5 \%$ to $5 \%$ of patients with $0.2 \%$ to $3 \%$ of patients developing mediastinitis [2,3]. Sternal dehiscence is the process of separation of the bony sternum, which often is accompanied by media stinitis (infection of the deep soft tissues). In thoracic and trunk reconstruction, plastic surgeons play a crucial role in addressing wound healing issues and reconstructive techniques of the chest wall. The management of chest wall reconstruction due to sternal dehiscence has evolved considerably over the past half century.

Theoretically autogenous bone tissue is preferable because it allows immediate restoration of a rigid surface without anyproblem of biological tolerance, achieving a sort of restitution ad integrum. Usually autologous bone grafts are harvested from the rib cage or from the iliac crest and further materials (autogenousor prosthetic) are required for the stabilization to be acceptable $[4,5]$. Several disadvantages limit the use of such osteoplasty: extensive surgical trauma, possible inadequacy of autologousmaterial, and heavier ventilatory functional damage if a rib graft is used.

Microsurgical techniques are extremely useful for reconstruction of complicated, composite chest wall defects. They are indicated when regional pedicled flap options are unavailable or inadequate. Microsurgical freetissue transfer of tissues allows great freedom in reconstruction of chest wall defects and has many advantages, compared to pedicled flaps and/or prosthetic materials [6]. The use of the free flap obviates concerns about compromised vascular supply, especially in coverage of large defects. Furthermore, constraints in positioning or placement of the tissue flap are avoided, as pedicle attachments are freed. The potential risks of microvascular surgery and free-tissue transfers are well known. Despite the inherent risk of vascular thrombosis and flap failure, successful microvascular anastomosis of vessels, a reliable and more robust vascular supply of the flap results, ultimately providing the best vascularized coverage of the defect [6]. In the selected group of patients with extensive resections of the chest wall for tumor the use of microsurgical techniques helps to transfer tissue from distant sites with good blood supply. After all local options are exhausted, these techniques provide reliable flaps to cover even the largest full-thickness chest wall defects.

Presented cases highlight the use of fibula free flap for a complex full-thickness anterior chest wall reconstruction.

\section{References}

[1] P. G. Arnold and P. C. Pairolero, "Chest-Wall Reconstruction: An Account of 500 Consecutive Patients,” Plastic and Reconstructive Surgery, Vol. 98, No. 5, 1996, pp. 804-810.

http://dx.doi.org/10.1097/00006534-199610000-00008

[2] C. Schimmer, S. P. Sommer, M. Bensch and R. Leyh, "Primary Treatment of Deep Sternal Wound Infection after Cardiac Surgery: A Survey of German Heart Surgery Centers,” Interactive CardioVasc Thoracic Surgery, Vol. 6, No. 6, 2007, pp. 708-711. http://dx.doi.org/10.1510/icvts.2007.164004

[3] V. A. Olbrecht, C. J. Barreiro, P. N. Bonde, J. A. Williams, W. A. Baumgartner, V. L. Gott and J. V. Conte, "Clinical Outcomes of Noninfectious Sternal Dehiscence after Median Sternotomy," The Annals of Thoracic Surgery, Vol. 82, No. 3, 2006, pp. 902-907. http://dx.doi.org/10.1016/j.athoracsur.2006.04.058

[4] J. E. Sherman, A. Salzberg, N. M. Raskin and E. J. Beattie, "Chest Wall Stabilization Using Plate Fixation," The Annals of Thoracic Surgery, Vol. 46, No. 4, 1988, pp. 467-469. http://dx.doi.org/10.1016/S0003-4975(10)64670-X

[5] J. H. Beggs and D. M. McCoy, "Reconstruction of the Sternum and Anterior Chest Wall Using Autologous Tissues,” Southern Medical Journal, Vol. 84, No. 5, 1991, pp. 655-656.

[6] P. G. Cordeiro, E. Santamaria and D. Hidalgo, "The Role of Microsurgery in Reconstruction of Oncologic Chest Wall Defects,” Plastic and Reconstructive Surgery, Vol. 108, No. 7, 2001, pp. 1924-1930. http://dx.doi.org/10.1097/00006534-200112000-00012 